\title{
Portugal : le malaise des enseignants
}

\section{Rui Canário}

\section{OpenEdition \\ Journals}

Édition électronique

URL : http://journals.openedition.org/ries/479

DOI : $10.4000 /$ ries.479

ISSN : 2261-4265

Éditeur

Centre international d'études pédagogiques

Édition imprimée

Date de publication : 1 avril 2009

Pagination : 16-18

ISSN : $1254-4590$

Référence électronique

Rui Canário, "Portugal : le malaise des enseignants », Revue internationale d'éducation de Sèvres [En ligne], 50 | avril 2009, mis en ligne le 01 avril 2012, consulté le 03 mai 2019. URL : http:// journals.openedition.org/ries/479; DOI : 10.4000/ries.479

Ce document a été généré automatiquement le 3 mai 2019.

(C) Tous droits réservés 


\title{
Le point sur...
}

\section{Portugal : le malaise des enseignants}

\author{
Rui Canário
}

1 L'année de 2008 a été au Portugal, pour les enseignants de l'enseignement de base et de l'enseignement secondaire, l'année de tous les mécontentements. Quelques faits sont suffisants pour comprendre soit la gravité, soit l'ampleur de ce malaise subi par une classe professionnelle dans sa totalité. Pendant l'année, par deux fois, les enseignants, mobilisés par les différents syndicats mais aussi dans le cadre de mouvements spontanés et autonomes, ont défilé sur le plus grand boulevard de Lisbonne, appelé, coïncidence heureuse, l'avenue de la Liberté. La première manifestation a eu lieu le 8mars et a réuni cent mille personnes, c'est à dire les deux tiers des professeurs de tout le pays. Cette réussite a constitué une énorme surprise pour tout le monde, syndicats inclus, car c'était la première fois que se rassemblaient autant d'enseignants venus, dans des conditions difficiles, de tous les coins du pays. Pourtant, la surprise a été encore plus grande quand, sous la pression de la base, la classe enseignante est sortie une deuxième fois, dans les rues de Lisbonne, le 8 novembre dernier. Environ cent vingt mille personnes ont défilé, soit les trois quarts des enseignants. Les deux manifestations ont eu lieu un samedi et le nombre des manifestants a été confirmé, de façon convergente, par plusieurs observateurs autonomes, et notamment la presse écrite, la radio et la télévision. Par la suite, le 3 décembre, les enseignants ont fait une grève au niveau national, dont le taux d'adhésion a été supérieur à $90 \%$. Une deuxième grève a eu lieu le 9 janvier 2009, avec une adhésion équivalente. La lutte des enseignants se poursuit sous de nouvelles formes, malgré une certaine érosion du mouvement, face à la rigidité des positions du gouvernement. Le mécontentement reste intense.

2 Face à des évènements inédits et totalement inattendus, un observateur externe peut légitimement chercher à connaître les raisons d'une telle démonstration de colère de la part d'une classe professionnelle traditionnellement peu combative et fragmentée par des tendances syndicales et politiques diverses. La réponse est relativement simple : ce sont les mesures prises par le ministère de l'Éducation et une action politique très peu raisonnable qui ont permis de créer cette ambiance de révolte qui traverse les 
établissements scolaires. En effet, le gouvernement a touché, avec ses reformes, à trois points clés, très sensibles pour les enseignants : premièrement, une nouvelle loi sur la gestion des établissements, laquelle réintroduit la figure du directeur (abolie depuis la révolution du 25 avril 1974) et limite fortement la tradition de fonctionnement démocratique des établissements; deuxièmement, une division et une hiérarchie de la carrière des enseignants qui créent, de façon perçue comme arbitraire et bureaucratique, une tranche de professeurs "titulaires" qui seront les seuls à pouvoir atteindre le sommet; enfin, la création d'un système d'évaluation individuelle des enseignants, qui a été le déclencheur de la contestation.

Ce système d'évaluation peut, de façon très concise, être caractérisé comme suit : on part $\mathrm{du}$ postulat selon lequel les enseignants sont coupables de tout ce qui va mal dans le système scolaire ; il s'agit d'un processus extrêmement lourd et bureaucratique qui tend à paralyser la vie des établissements ; il est basé sur une vision atomisée et individuelle du fonctionnement de l'établissement, en ignorant les dimensions collectives du travail enseignant, aussi bien que la totalité systémique qu'est l'établissement scolaire; il est centré sur une évaluation qui rend totalement subalternes les dimensions formatives et qui devient punitif, car elle a des conséquences sur la progression dans la nouvelle carrière en limitant l'accès aux niveaux supérieurs. Enfin, ce modèle d'évaluation est perçu, pour de "bonnes raisons ", comme très injuste car il est censé évaluer le mérite absolu de chacun et établit en même temps une limite pour l'attribution des mentions « excellent » $(5 \%)$ et « très bon » $(20 \%)$, ce qui empêche les trois quarts des enseignants de progresser jusqu'au sommet.

4 Tout ce cadre a comme toile de fond l'émergence d'un phénomène de chômage qui touche particulièrement les jeunes qui viennent d'être formés; la baisse réelle de la valeur des salaires ; l'augmentation de la durée de l'horaire de travail ; des conditions de plus en plus difficiles pour exercer le métier, surtout dans les zones urbaines plus sensibles du point de vue social. Toutes les conditions sont donc créées pour « inviter " les enseignants à choisir des stratégies collectives pour affronter les mesures prises. Celles-ci expriment à la fois : un processus de centralisation qui diminue l'autonomie et augmente le contrôle ; une manière d'essayer de légitimer des politiques restrictives en matière de salaires des fonctionnaires.

5 Cette situation portugaise exprime des tendances qui, à différents degrés, marquent négativement l'exercice du métier enseignant dans une grande diversité de pays dans le monde. Ce qu'elle présente de singulier est l'ampleur du mouvement et du niveau de souffrance au travail qu'il révèle. Le quotidien des enseignants est devenu invivable. «Laissez-nous être professeurs! » est devenu le mot d'ordre le plus populaire et celui qui exprime le mieux cette angoisse collective. Ironiquement, on peut souligner la contradiction entre les mesures effectivement prises et la rhétorique exprimée à Lisbonne, lors de la tenue (en 2007) d'une Conférence ${ }^{1}$ consacrée au thème du «développement professionnel des enseignants». Dans cette conférence, l'éloge du métier enseignant comme facteur essentiel pour atteindre les objectifs définis dans la «stratégie de Lisbonne » a été un axe central. D'autre part, les conclusions ont retenu trois grands défis politiques : rendre la profession enseignante attractive; contrarier l'invasion de l'école par les problèmes sociaux; dépasser le paradoxe de la coexistence d'une prétendue autonomie avec des modalités de plus en plus dures de contrôle. Il nous faut redécouvrir les chemins qui puissent nous conduire à retrouver le plaisir d'enseigner. 


\section{NOTES}

1. Voir : PORTUGAL (2008). Portuguese Presidency of the Council of the European Union Conférence "Teacher professional development for the quality and equity of lifelong learning". Lisbonne, 27-28 septembre 2007.

\section{INDEX}

Index géographique : Portugal

Mots-clés : enseignant, évaluation

Keywords : teachers, teacher evaluation

Palabras claves : docente, evaluación del docente

\section{AUTEUR}

\section{RUI CANÁRIO}

Il dirige l'Unité de recherche et de développement en sciences de l'éducation de l'Université de Lisbonne, Portugal. Son enseignement et ses recherches portent sur la sociologie de l'éducation et de la formation des adultes. Il dirige depuis quatre ans un projet de recherche, financé par la FCT (Fondation pour la science et la technologie), sur les « politiques et pratiques de formation des adultes au Portugal depuis 1974 ». Il dirige également un projet de recherche luso-brésilien sur les « politiques de formation concernant les jeunes et adultes peu scolarisés au Portugal et au Brésil ", étude comparative basée sur un réseau qui regroupe des chercheurs de l'Université de Lisbonne et de plusieurs universités du Brésil. Il est membre du comité scientifique de plusieurs revues internationales. 